\title{
Efecto de los hidruros en el comportamiento mecánico de vainas de zircaloy de combustible nuclear en dirección anular ${ }^{(*)}$
}

\author{
M.A. Martin-Rengel*, J. Ruiz-Hervías ${ }^{(2) * *}$, F.J. Gómez**, L. Caballero** y A. Valiente**
}

\begin{abstract}
Resumen
En este artículo se ha analizado el efecto de los hidruros en el comportamiento mecánico de las vainas de circonio que se emplean para contener las pastillas de combustible nuclear. Dicho comportamiento se ha evaluado en la dirección anular de las vainas (la más solicitada en servicio), mediante ensayos de tracción en anillo sobre muestras con distintas concentraciones de hidrógeno, y se ha propuesto un modelo de elementos finitos en 3D para simular los ensayos. Los hidruros fueron introducidos en las muestras mediante una técnica de carga catódica de hidrógeno y posterior tratamiento térmico. Los resultados muestran que a las concentraciones ensayadas no existe una degradación significativa de las propiedades mecánicas en la dirección circunferencial, aunque los micromecanismos de rotura cambian con el contenido en hidrógeno de las muestras.
\end{abstract}

Palabras clave Vainas de combustible; Circonio; Hidruros; Ensayo de tracción en anillo; Propiedades mecánicas.

\section{Influence of hydrides on the mechanical behaviour of zircaloy nuclear cladding along the hoop direction}

\begin{abstract}
The effect of hydrides on the mechanical behaviour of the zirconium-alloy cladding employed as nuclear fuel barrier was studied in this paper. Ring tensile tests were performed on samples with different hydrogen concentrations and a $3 \mathrm{D}$ finite element model was developed to simulate the test. Hydrides were introduced by cathodic charging with hydrogen and subsequent thermal treatment of cladding samples. The tests show that there is not a significant degradation of the mechanical behaviour at the hydrogen concentrations tested, although the fracture micromechanisms depend on the hydrogen content.
\end{abstract}

Keywords

Fuel claddings; Zirconium; Hydrides; Ring tensile test; Mechanical properties.

\section{INTRODUCCIÓN}

Las pastillas cerámicas que forman el combustible nuclear, están contenidas en el interior de tubos de pared delgada, denominados vainas. Dichas vainas constituyen la primera barrera estructural para contener el combustible y los productos de fisión y deben mantener confinado este material durante el ciclo del combustible. Estas vainas se fabrican con aleaciones de circonio debido a sus excelentes propiedades mecánicas, buena resistencia a la corrosión y transparencia al flujo neutrónico. En condiciones de operación, la reacción de oxidación que se produce en la superficie exterior de las vainas (en contacto con agua o vapor de agua a $300^{\circ} \mathrm{C}$ ) produce hidrógeno. Dicho hidrógeno es absorbido por la aleación y se difunde en su interior, precipitando en forma de hidruros cuando se alcanza el límite de solubilidad. Si los hidruros se encuentran orientados desfavorablemente respecto a la tensión que soportan las vainas, éstas se fragilizan y su durabilidad puede disminuir ${ }^{[1-3]}$.Por esta razón es de suma importancia evaluar el efecto fragilizador en términos de la integridad estructural remanente de las vainas.

Durante distintas fases del ciclo del combustible, como durante el almacenamiento temporal en seco $^{[4]} \mathrm{y}$ en determinados accidentes $\left.{ }^{[5} \mathrm{y} 6\right]$, las vainas

(•) Trabajo recibido el día 28 de julio de 2008 y aceptado en su forma final el día 13 de marzo de 2009 .

* Consejo de Seguridad Nuclear (CSN); Justo Dorado 11, E-28040 Madrid, Spain; Tel: (+34) 913366685, Fax: (+34) 913366680, e-mail: mamartin@mater.upm.es

** Departamento de Ciencia de Materiales, UPM, E.T.S.I. Caminos, Canales y Puertos; Profesor Aranguren s/n, E-28040 Madrid, Spain; Tel: (+34) 913366685 , Fax: (+34) 913366680 , email: jr@mater.upm.es. 
soportan importantes solicitaciones mecánicas en dirección circunferencial. Dado que las propiedades mecánicas de estas aleaciones son anisótropas ${ }^{[7]}$, es preciso desarrollar un ensayo que permita la caracterización mecánica de las vainas en dirección circunferencial. Uno de los ensayos propuestos en la literatura es el ensayo de tracción en anillo, homologado para tubos de diámetros mucho mayores ${ }^{[8]}$ que el de las vainas de combustible nuclear. Se han hecho distintas propuestas para adaptar este ensayo al caso de las vainas de combustible nuclear, siendo una de las más importantes la realizada por Arsen ${ }^{[1]}$. Este ensayo se ha empleado para comparar el comportamiento de distintos materiales irradiados y tiene numerosas ventajas en dicha aplicación: las muestras son relativamente fáciles de mecanizar y se emplea muy poca cantidad de material. El problema reside en caracterizar de forma cuantitativa el comportamiento mecánico de las vainas en dirección circunferencial. Para ello es preciso determinar la tensión y la deformación local en el anillo durante el ensayo a partir de la fuerza aplicada y del desplazamiento de la máquina de ensayos ${ }^{[1]}$

En este trabajo se han realizado ensayos de tracción en anillo en muestras de vainas de combustible nuclear con distintas concentraciones de hidrógeno (0-2000 ppm) y se ha llevado a cabo un estudio fractográfico de las muestras rotas. Se ha desarrollado una técnica experimental para introducir hidrógeno de forma controlada mediante carga catódica. Una vez cargadas con hidrógeno, las muestras se someten a un tratamiento térmico para generar la población de hidruros deseada.

Además, se ha puesto a punto un modelo de elementos finitos en 3D para simular el ensayo y calcular la tensión y la deformación local en la sección reducida de las muestras. Los resultados muestran que el comportamiento mecánico en la dirección circunferencial no depende de forma significativa del contenido de hidrógeno. Sin embargo, el aspecto que presenta la superficie de fractura de las muestras es distinto según la concentración de hidrógeno en las mismas, lo que sugiere distintos mecanismos de rotura.

\section{EXPERIMENTAL}

\subsection{Material}

En este trabajo se han empleado vainas de combustible nuclear para reactores de agua a presión (PWR), con un diámetro exterior de $9,5 \mathrm{~mm}$ y un espesor de pared de 0,56 mm. La composición química del material se recoge en la tabla I:
Tabla I. Composición química de las vainas de circonio estudiadas

Table I. Chemical composition of the zirconium cladding employed

\begin{tabular}{cccccc}
\hline Sn & $\mathrm{Fe}$ & $\mathrm{Cr}$ & $\mathrm{O}$ & $\mathrm{Nb}$ & $\mathrm{Zr}$ \\
\hline 1,0 & 0,01 & 0,008 & 0,01 & 1,0 & Bal. \\
\hline
\end{tabular}

Las propiedades mecánicas en la dirección axial se obtuvieron mediante ensayos de tracción en probetas cilíndricas ( $120 \mathrm{~mm}$ de longitud) cortadas a partir de las vainas. Los ensayos se realizaron en control de desplazamiento a una velocidad de $0,3 \mathrm{~mm} / \mathrm{min}$. Las propiedades mecánicas obtenidas (media de tres ensayos) se presentan en la tabla II.

Las probetas para los ensayos de tracción anular se fabricaron a partir de vainas cortadas en anillos de 5 $\mathrm{mm}$ de altura sobre las que se mecanizaron dos secciones reducidas simétricas de $2 \mathrm{~mm}$ de altura, como puede verse en la figura 1 .

El sistema de carga se diseñó, específicamente, para este trabajo y consiste en dos vigas semicilíndricas en contacto con la superficie interior de las probetas, que se van separando de forma progresiva según se desplaza el pistón de la máquina de ensayos. En el interior del anillo se coloca un centrador con el objetivo de que las secciones reducidas de la muestra no entren en flexión ${ }^{[8]}$ al principio del ensayo. En la figura 2 se muestra el dispositivo experimental. El ensayo se realizó en control de desplazamiento a una velocidad de $0,3 \mathrm{~mm} / \mathrm{min}$ y se midió el desplazamiento del pistón y la carga aplicada a las vigas (célula de carga de $10 \mathrm{kN}$ ).

\subsection{Carga con hidrógeno}

Se ha desarrollado una técnica para introducir cantidades controladas de hidrógeno en las probetas. Esta técnica consiste en la carga catódica de las

Tabla II. Propiedades mecánicas de las vainas de circonio en la dirección axial

Table II. Mechanical properties of the zirconium cladding in the axial direction

\begin{tabular}{cccc}
\hline$E(\mathrm{GPa})$ & $\sigma_{\max }(\mathrm{MPa})$ & $\sigma_{0.2}(\mathrm{MPa})$ & $\varepsilon_{\max }(\%)$ \\
\hline 90 & 805 & 610 & 7,4 \\
\hline
\end{tabular}



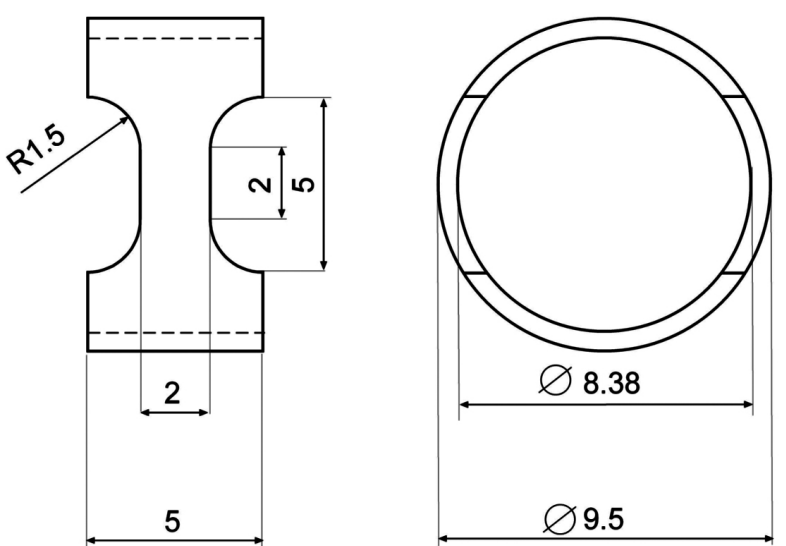

Figura 1. Dimensiones de las probetas de tracción en anillo.

Figure 1. Dimensions of the ring tensile test samples.

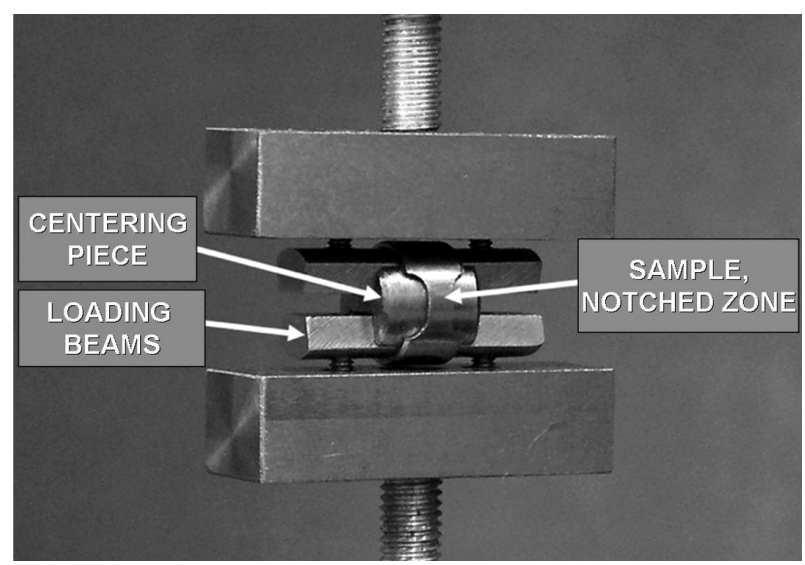

Figura 2. Dispositivo experimental utilizado en los ensayos de tracción en anillo.

\section{Figure 2. Experimental loading system employed in the ring tensile test.}

muestras en disolución acuosa de $\mathrm{KOH}$. La probeta de circonio se utilizó como cátodo, mientras que el ánodo era un hilo de platino enrollado a su alrededor. La carga se realizó manteniendo la densidad de corriente constante, con valores entre 0,25 y $2,15 \mathrm{~A} / \mathrm{cm}^{2}$ y tiempos de carga entre 3 y 25 h, en función de la cantidad de hidrógeno a introducir en la probeta. Una vez cargadas, las muestras fueron tratadas térmicamente en atmósfera de argon. El tratamiento consistió en una calentamiento a $723 \mathrm{~K}$ durante $7 \mathrm{~h}$, seguido de un enfriamiento lento, a una velocidad de $1,2 \mathrm{~K} / \mathrm{min}$. Como resultado de este tratamiento se obtuvieron hidruros con una longitud compren- dida entre 45 y $125 \mu \mathrm{m}$ distribuidos de forma bastante homogénea en las muestras.

La concentración de hidrógeno en las muestras se midió con un analizador HORIBA JOBIN-YVON EMGA-621W. El método de medida consiste en la extracción, a alta temperatura, del hidrógeno que es recogido por un gas portador, argon. Mediante la medida de la conductividad térmica de la mezcla de gases se determina la cantidad de hidrógeno presente en la muestra.

La morfología y distribución de los hidruros se estudió con técnicas metalográficas. Las probetas fueron embutidas en resina epoxi y pulidas con suspensión de alúmina de $1 \mu \mathrm{m}$. El acabado se obtuvo puliendo las muestras con una mezcla de sílice coloidal $(0,05 \mu \mathrm{m}), \mathrm{H}_{2} \mathrm{O}_{2}(30 \% \mathrm{vol})$ y $\mathrm{HF}(40 \% \mathrm{p})$ en proporción (50:10:1 en volumen). Para el revelado de hidruros las muestras fueron atacadas durante $90 \mathrm{~s}$ con una disolución de $\mathrm{HNO}_{3}$ (69\% p), ácido láctico (95\% p) y HF (40 \% p) en proporción (48,5: 48,5: 3 en peso).

\subsection{Modelo de elementos finitos}

Se ha desarrollado un modelo de elementos finitos en 3D con el programa ABAQUS v 6.3 para simular el ensayo de tracción en anillo y caracterizar las propiedades mecánicas de las vainas en la dirección circunferencial. Se empleó una malla tridimensional considerando sólo un octavo de la probeta debido a las simetrías geométricas y de carga. Los elementos empleados fueron paralelepípedos bilineales con 21 nodos. El tamaño de los elementos en la sección reducida fue de unas $40 \mu \mathrm{m}$. Las cargas y las condiciones de contorno se simularon como un problema de contacto entre sólidos, considerando dos superficies rígidas para aplicar la carga (modelización de las vigas de carga) y para limitar el movimiento del anillo en dirección perpendicular (modelización de la constricción del centrador). Por tanto, se trata de un problema de contacto doble con rozamiento de tipo Coulomb. En los cálculos se consideró no linealidad geométrica, en todos los casos. El comportamiento del material se consideró elastoplástico y transversalmente isótropo, con criterio de plastificación de Hill. Se ha empleado un coeficiente de rozamiento de 0,125 (como se propone en la literatura ${ }^{[1]}$ y y las constantes plásticas correspondientes a la dirección axial recogidas en la tabla II. Se ha realizado un estudio paramétrico del efecto de las constantes plásticas, del coeficiente de rozamiento y de las holguras entre la muestra y el dispositivo de carga. Para un valor específico del coeficiente de rozamiento se ha propuesto un valor de la tensión de plastificación y una curva uniaxial 
tensión-deformación plástica, con objeto de explicar la curva experimental carga-desplazamiento de las vigas de carga del sistema de ensayos.

\section{RESULTADOSY DISCUSIÓN}

El método de carga catódica de hidrógeno da lugar a una población de hidruros de 45 a $125 \mu \mathrm{m}$ de longitud distribuidos a lo largo de la sección transversal de las muestras, como puede verse en la figura 3. Los hidruros son las líneas oscuras observadas sobre la superficie clara de circonio.

Para los ensayos de tracción en anillo se cargaron probetas con distintos contenidos de hidrógeno: 150 , $250,500,1.000$ y 2.000 ppm

Las curvas experimentales carga-desplazamiento fueron corregidas siguiendo el método propuesto por otros autores ${ }^{[8]}$. El desplazamiento del pistón tiene dos contribuciones: una, correspondiente al dispositivo de carga y, otra, correspondiente a la deformación de la muestra. Para calibrar el desplazamiento causado por el dispositivo de carga se realizaron tres ensayos con un anillo rígido de acero de 3,5 $\mathrm{mm}$ de espesor de pared, mucho mayor que el de las muestras, 0,5 mm. Como resultado de la calibración se obtuvo la siguiente ecuación que proporciona el desplazamiento debido a la deformación de la muestra en función del desplazamiento del pistón y de la fuerza aplicada:

$$
d=d_{t}-F / 6900
$$

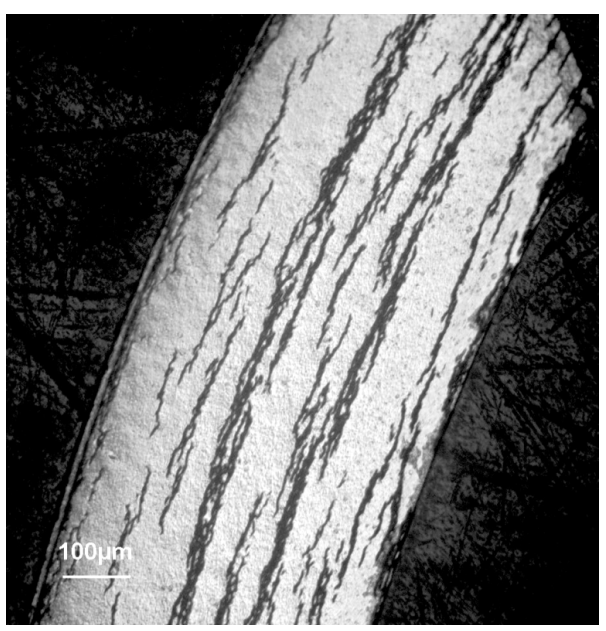

Figura 3. Distribución de hidruros en una probeta de tracción anular con 500 ppm de hidrógeno.

Figure 3. Hydride distribution in a ring sample with 500 ppm hydrogen. donde, $d_{t}$ es el desplazamiento del pistón, $F$ es la fuerza aplicada y $d$ es el desplazamiento corregido (debido solamente a la deformación de la muestra).

Las curvas carga-desplazamiento (corregidas como se ha explicado anteriormente) para las distintas concentraciones de hidrógeno ensayadas se muestran en las figuras 4-9. Todas las curvas presentan un comportamiento similar, independientemente de la concentración de hidrógeno en la muestra. Hay pequeñas variaciones en la carga máxima, que parece aumentar en las muestras con 150 y 250 ppm con respecto a las muestras en estado de recepción. Para

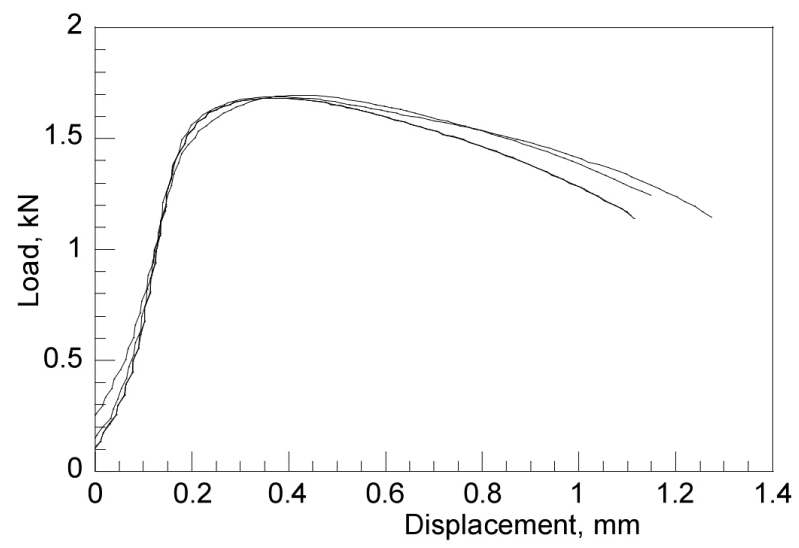

Figura 4. Registro carga frente a desplazamiento en probetas de tracción en anillo en "estado de recepción" (sin hidrógeno).

Figure 4. Load vs. displacement plot in "asreceived" ring tensile samples (without hydrogen).

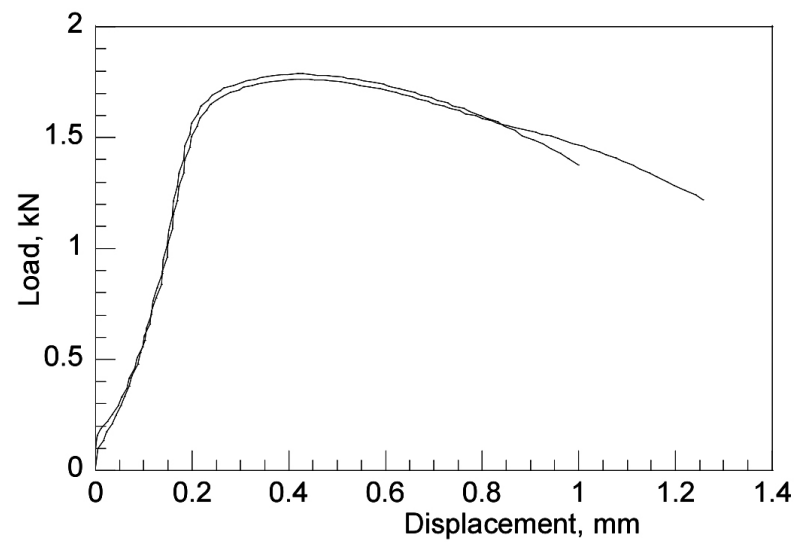

Figura 5. Registro carga frente a desplazamiento en probetas de tracción en anillo (150 ppm de hidrógeno).

Figure 5. Load vs. displacement plot in ring tensile samples (150 ppm hydrogen). 


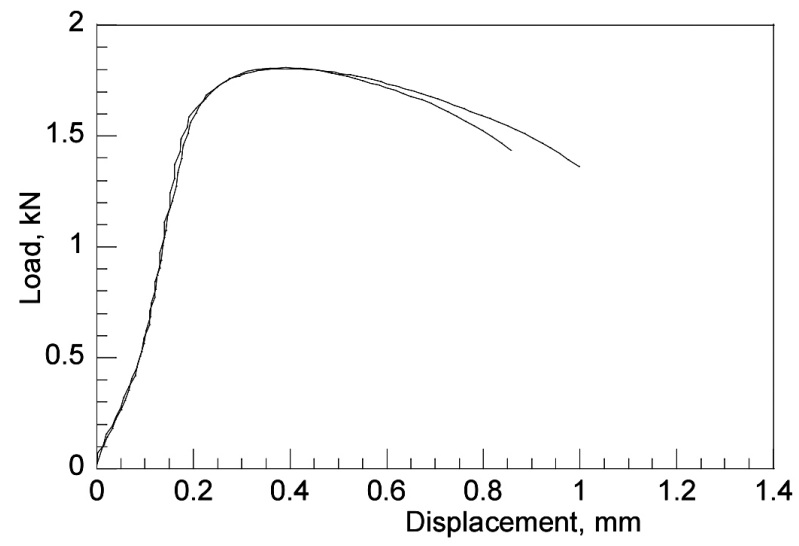

Figura 6. Registro carga frente a desplazamiento en probetas de tracción en anillo $(250$ ppm de hidrógeno).

Figure 6. Load vs. Displacement plot in ring tensile samples (250 ppm hydrogen).

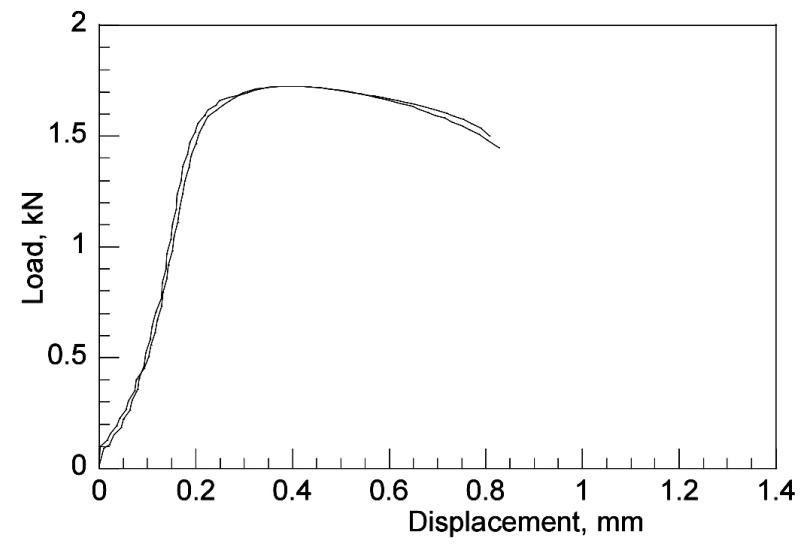

Figura 7. Registro carga frente a desplazamiento en probetas de tracción en anillo (500 ppm de hidrógeno).

Figure 7. Load vs. displacement plot in ring tensile samples (500 ppm hydrogen).

las muestras con altas concentraciones de hidrógeno ensayadas ( 1.000 y 2.000 ppm), la carga máxima tiende a disminuir respecto de los valores encontrados a $250 \mathrm{ppm}$.

Los resultados de la simulación por elementos finitos se presentan en las figuras 10 y 11 para una muestra en estado de recepción y otra con 250 ppm de hidrógeno, respectivamente. Puede verse que existe una buena coincidencia con los datos experimentales en ambos casos:

La evolución del límite elástico al 0,2%, obtenido a partir de las simulaciones numéricas, con el

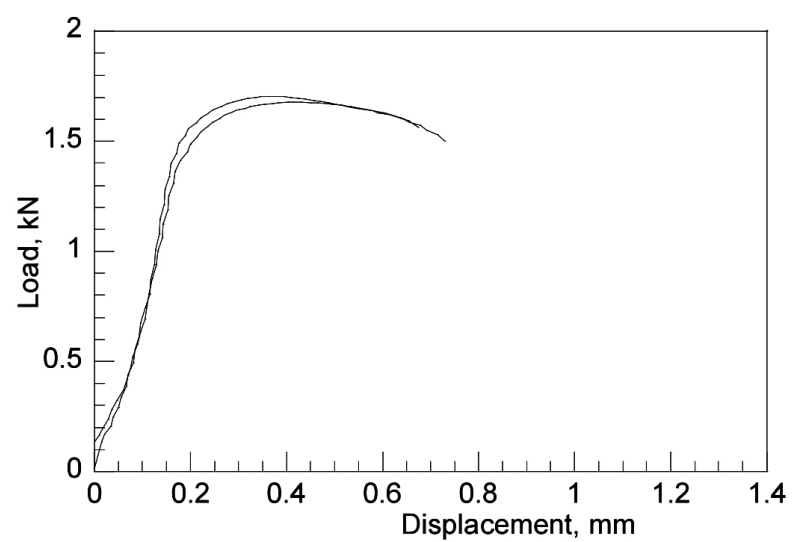

Figura 8. Registro carga frente a desplazamiento en probetas de tracción en anillo (1.000 ppm de hidrógeno).

Figure 8. Load vs. displacement plot in ring tensile samples (1,000 ppm hydrogen).

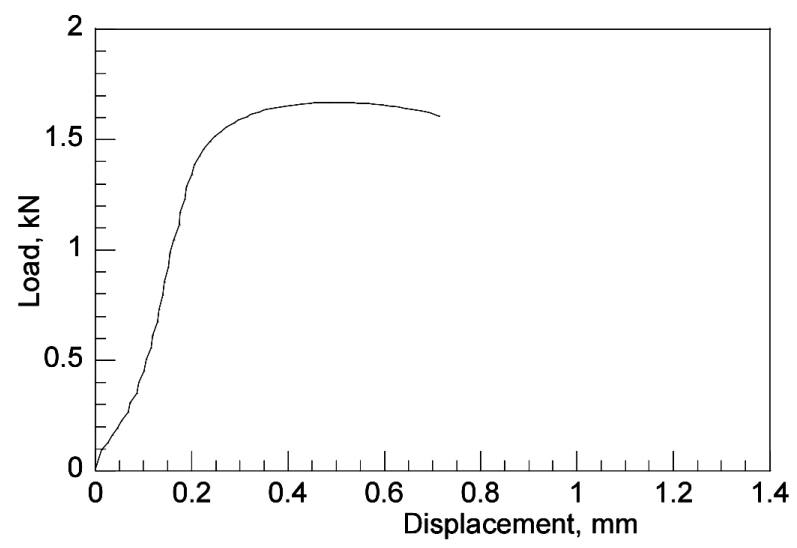

Figura 9. Registro carga frente a desplazamiento en probetas de tracción en anillo $(2.000$ ppm de hidrógeno).

Figure 9. Load vs. displacement plot in ring tensile samples (2,000 ppm hydrogen).

contenido de hidrógeno se presenta en la figura 12. Como sucede para carga máxima, existe un pequeño incremento para el límite elástico entre 150 y 250 ppm (del 6,7 \% para el caso de 250 ppm) y un descenso para contenidos de hidrógeno mayores, llegando una reducción del $11 \%$ para 2.000 ppm. Estos resultados concuerdan con los obtenidos en la literatu$\mathrm{ra}^{[1]}$ para vainas de distintas aleaciones de circonio a temperatura ambiente. Además, el límite elástico en la dirección circunferencial es mayor que el obtenido para la dirección axial (tabla II), lo que coincide con los resultados de otros autores ${ }^{[7]}$. 


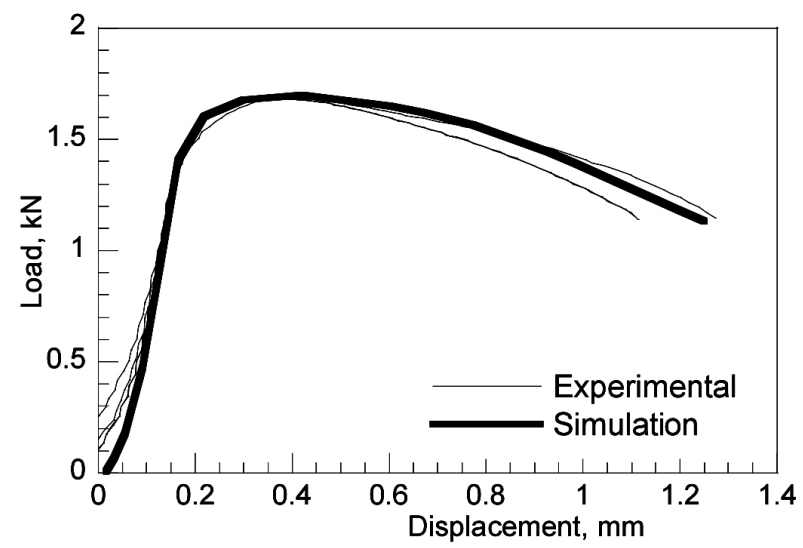

Figura 10. Registros carga-desplazamiento experimentales y simulados en probetas de tracción en anillo en "estado de recepción" (sin hidrógeno).

Figure 10. Experimental and simulated load vs. Displacement plots in "as-received" ring tensile samples (without hydrogen).

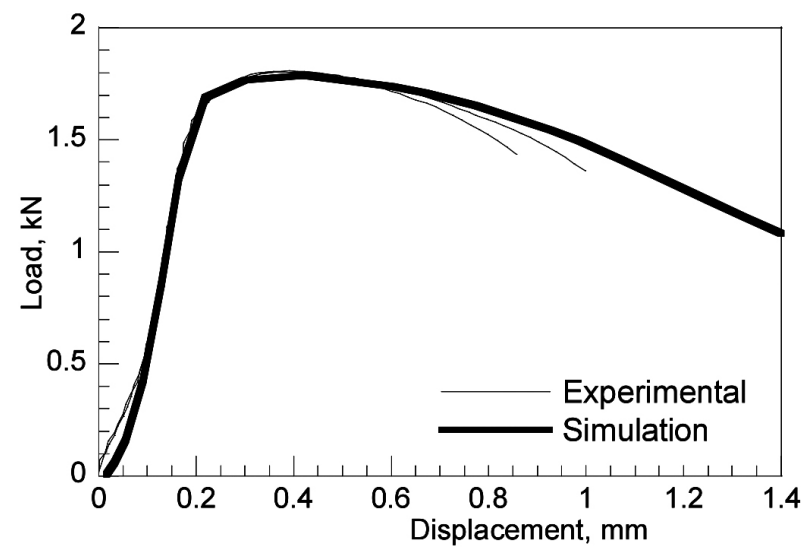

Figura 11. Registros carga-desplazamiento experimentales y simulados en probetas de tracción en anillo (250 ppm de hidrógeno).

Figure 11. Experimental and simulated load vs. Displacement plots in ring tensile samples $(250$ ppm hydrogen).

Las superficies de fractura de las probetas rotas se analizaron en el microscopio electrónico de barrido. En las probetas en estado de recepción, la rotura macroscópica se produce a $45^{\circ}$ respecto de la dirección anular, como se puede ver en la figura 13, localizándose en la zona central de la sección reducida de la probeta. Se aprecia una importante estricción de la muestra en las zonas próximas a la rotura. En la figura 14 puede observarse un detalle representativo de la

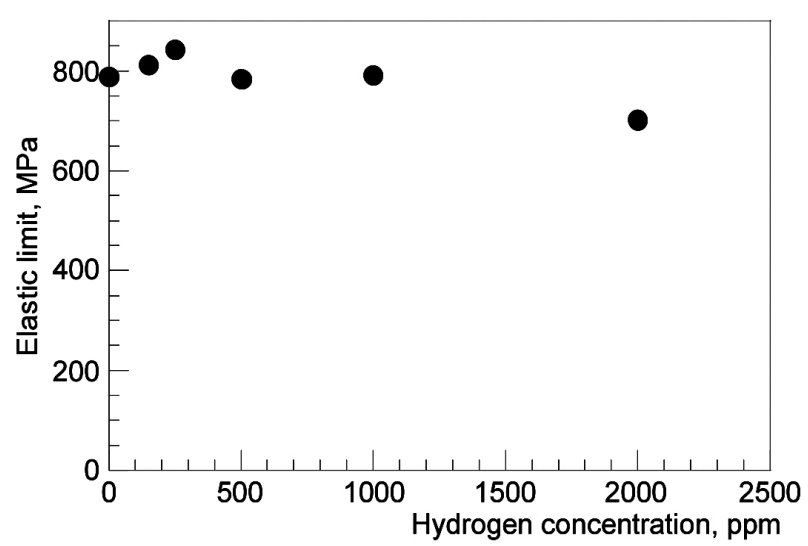

Figura 12. Evolución del límite elástico en dirección circunferencial (calculado a partir de las simulaciones numéricas) en función del contenido de hidrógeno.

Figure 12. Elastic limit in hoop direction (calculated from numerical simulations) in versus hydrogen concentration.

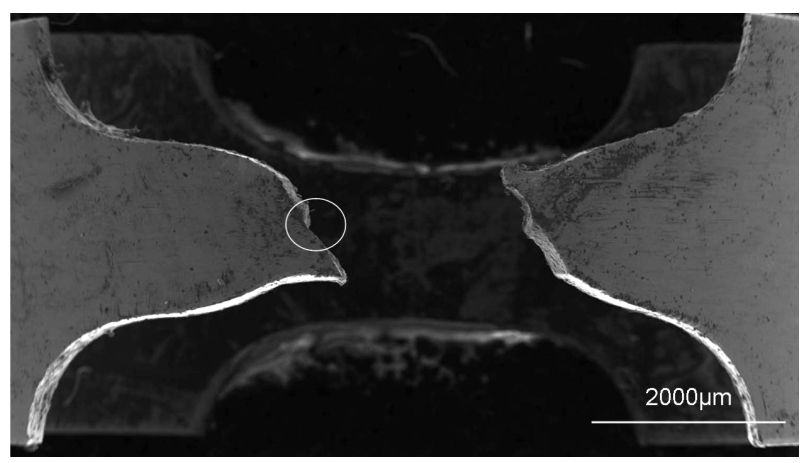

Figura 13. Probeta en "estado de recepción" (sin hidrógeno) sometida a tracción anular vista en el microscopio electrónico de barrido.

Figure 13. SEM image of an "as-received" (withouth hydrogen) ring tensile sample after testing.

superficie de rotura en estado de recepción. Esta superficie se encuentra en la zona rodeada con una circunferencia en la figura 13. Puede apreciarse, claramente, que en esta probeta la rotura ha sucedido por un mecanismo de nucleación, crecimiento y coalescencia de huecos; es decir, un mecanismo asociado a ductilidad a nivel macroscópico.

El aspecto macroscópico de la fractura en las muestras cargadas con 2.000 ppm de hidrógeno se muestra en la figura 15. En este caso, la rotura sigue, globalmente, la normal a la dirección de solicitación y está localizada en uno de los extremos de la sección 


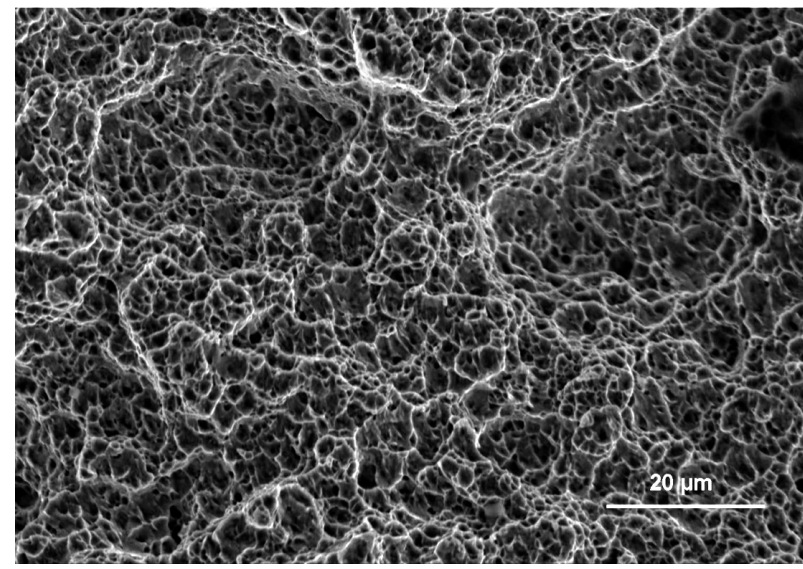

Figura 14. Detalle de la figura 13 correspondiente a una probeta en estado de recepción (sin hidrógeno) vista en el microscopio electrónico de barrido.

Figure 14. Detail of the figure 13 corresponding to an "as received" (without hydrogen) ring tensile sample.

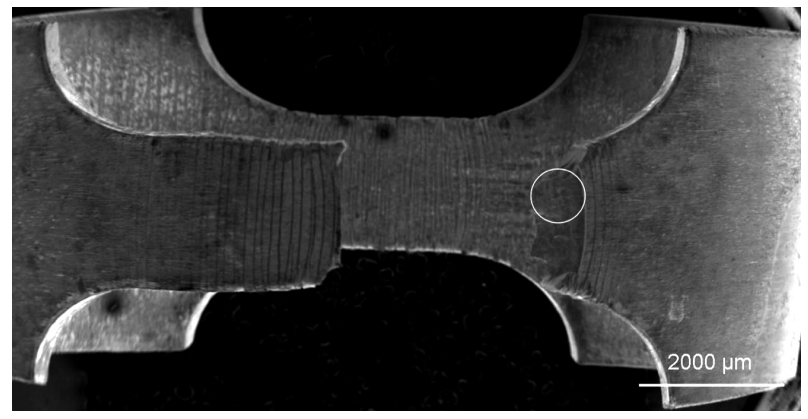

Figura 15. Probeta cargada con 2.000 ppm de hidrógeno sometida a tracción anular vista en el microscopio electrónico de barrido.

\section{Figure 15. SEM image of a ring tensile sample with 2,000 ppm hydrogen alter testing.}

reducida de la probeta. Se aprecian numerosas grietas paralelas que atraviesan verticalmente la sección reducida, lo que ocurre tanto para la cara rota como para la cara opuesta. La figura 16 recoge un detalle representativo de la superficie de rotura de la probeta ensayada con 2.000 ppm de hidrógeno. Esta superficie se localiza en la zona rodeada con una circunferencia en la figura 15. Pueden observarse bandas o terrazas en las que se ven superficies facetadas con orientaciones locales que sugieren una posible relación con planos cristalográficos, lo que indica un mecanismo de cuasiclivaje. Las bandas descritas se encuentran separadas por franjas en las que se aprecian desgarramientos dúctiles.

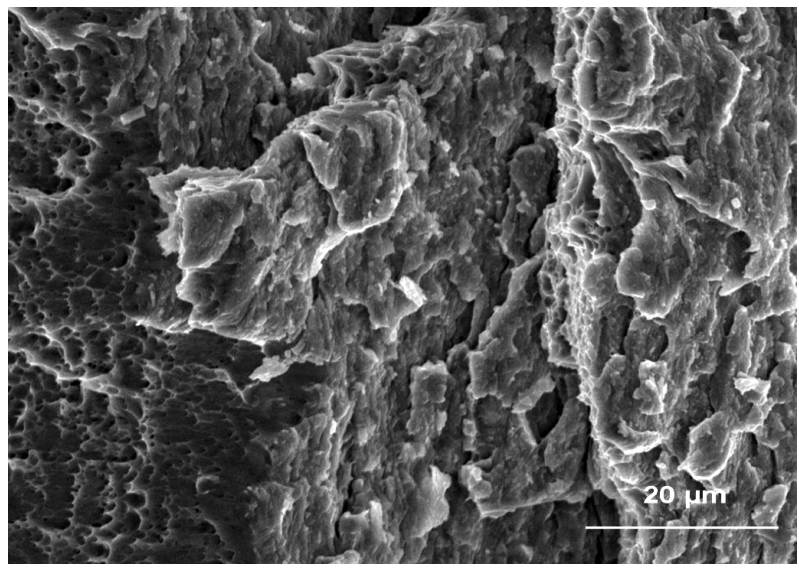

Figura 16. Detalle de la figura 15 correspondiente a una probeta cargada con 2.000 ppm de hidrógeno sometida a tracción anular vista en el microscopio electrónico de barrido.

Figure 16. "Detail of the figure 15 corresponding to a ring tensile sample with $2,000 \mathrm{ppm}$ hydrogen.

\section{CONCLUSIONES}

Se ha desarrollado un proceso de laboratorio para crear una población homogénea de hidruros en muestras de vainas de combustible nuclear. El proceso está basado en la carga catódica de hidrógeno seguida de un tratamiento térmico de homogeneización y es muy reproducible. Con este procedimiento se ha podido introducir hasta $2.000 \mathrm{ppm}$ de hidrógeno en las muestras. Esto permite simular en el laboratorio las condiciones de daño asociadas a los hidruros generados durante las distintas fases del ciclo del combustible, evitando trabajar con materiales irradiados.

El comportamiento mecánico de las vainas de combustible nuclear en dirección circunferencial se ha evaluado mediante ensayos de tracción en anillo. Los resultados experimentales muestran que a las concentraciones de hidrógeno ensayadas (hasta 2.000 ppm), no hay una degradación significativa del comportamiento mecánico a temperatura ambiente. Sin embargo, los micromecanismos de rotura cambian sustancialmente al aumentar el contenido de hidrógeno. En las muestras en estado de recepción (sin hidrógeno) se aprecia una notable estricción y domina la nucleación y coalescencia de huecos, mecanismos asociados a la ductilidad macroscópica. Sin embargo, en las muestras cargadas con 2.000 ppm de hidrógeno, prácticamente no hay estricción y la superficie de rotura presenta rasgos típicos de cuasi-clivaje, lo que está asociado a un comportamiento macroscópico frágil. 
El modelo de elementos finitos en 3D desarrollado en este trabajo reproduce de forma precisa los resultados experimentales obtenidos. Esto permite confiar en los valores de tensión y deformación local calculados en la sección reducida de las muestras. El límite elástico obtenido a partir de la simulación numérica cambia muy poco con el contenido de hidrógeno y su valor es mayor que el obtenido en dirección axial a partir de los ensayos mecánicos.

\section{Agradecimientos}

Los autores quieren agradecer la financiación recibida del Ministerio de Educación y Ciencia (proyecto ENE 2005-06478/CON) y a ENUSA, ENRESA y CSN su colaboración en este proyecto. Uno de los autores (M.A. Martín) desea agradecer al CSN la beca concedida para la realización del proyecto. El presente trabajo ha sido desarrollado en el marco de los proyectos DUMEINPA, financiado por la comunidad de Madrid, y SEDUREC, del proyecto nacional de investigación CONSOLIDER-INGENIO 2010.

\section{REFERENCIAS}

[1] S. Arséne, Ph.D. dissertation, École Centrale, Paris (1997).

[2] M. Grange, Ph.D. dissertation, École des Mines de Paris (1998).

[3] G. Bertolino, G. Meyer y J. Perez-Ipiña, J.Nucl. Mater. 320 (2003) 272-279.

[4] J.Y.R. Rashid y A.J. Machiels, ICEM05, 2005.

[5] J. Desquines, B. Cazalis, C. Bernaudat, C. Poussard, X. Averty y P.Yvon J. ASTM Int. 2 (2005).

[6] B. Cazalis, J. Desquines, C. Possard, M. Petit, Y. Monerie, C. Bernaudat, P.Yvon y X.Averty, Nucl. Technol. 157 (2007) 215-229.

[7] K. Linga y I. Charit, Prog.Nucl. Energ. 48 (2006) 325-359.

[8] S. Arséne y J. Bai, J.Test. Eval. 26 (1998) 26-30. 